\section{RECEST: A program package for the estimation of parameters of dynamic linear systems}

\author{
H. W. BRAAKMAN and F. J. J. van BUSSEL
}

Tilburg University, Hogeschoollaan 225, Tilburg, The Netherlands

In the analysis of behavioral data, most experimental designs are directed toward revealing static characteristics of the studied system. A large number of techniques are available to assess the mean effects of certain independent variables on certain other dependent variables. Statistical techniques are used to enable deductions about the likelihood of various possible outcomes of observations (Hays, 1973). The mathematical models that are then based on the set of measurements are static in nature. The dynamic characteristics are not often considered. When, for instance, a random variable is to be described, only the moments of the distribution (mean, variance, skewness) are considered. Dynamic properties (autocorrelations) are not described.

This apparent lack of interest in the dynamic properties of variables is reflected in the tendency to study only static properties of behavior. One of the reasons for this situation could be the fact that analytical techniques for the study of dynamic effects are not as readily available as those for the more common statistical analysis. The program package described in this paper enables the estimation of parameters of dynamic systems. Currently, its use is restricted to single-input/singleoutput situations.

The package includes two parameter estimation techniques, one recursive and one nonrecursive. The nonrecursive method uses all input and output samples to produce one set of estimates for the parameters. In recursive estimation, each pair of data points (one input and one output sample) is used to update the estimates for the parameters of the transfer function. Therefore, at each moment in time, an estimate of the parameters is available. The recursive method can be used to detect changes in the behavior of the system if those are reflected in the transfer function, for instance, sudden changes in strategy or insight of the subject whose behavior is to be modeled.

This package has been applied to data from an experiment in which subjects had to predict future values of time series. A transfer function for the prediction process was formulated (van Bussel, in press). For this kind of application, the dynamic analysis is rather obvious. In operant conditioning, decision making, and sequential motor tasks (e.g., reaction time studies), dynamic properties of the subject's behavior are also important.

Description of the Model. It is assumed that the relation between the input $u($.$) and the output signal$ $y($.) can be described by a linear dynamic model of the ARMA type:

$$
\begin{aligned}
y(t)= & -a_{1} y(t-1)-\ldots-a_{n_{a}} y\left(t-n_{a}\right) \\
& +b_{0} u(t)+b_{1} u(t-1)+\ldots \\
& +b_{n_{b}} u\left(t-n_{b}\right)+e(t),
\end{aligned}
$$

where $n_{a}$ and $n_{b}$ represent the orders of the autoregressive part and the moving average part, respectively. The equation noise e(.) is generated by an unknown noise signal $\xi($.$) through a similar model:$

$$
\begin{aligned}
e(t)= & \xi(t)+c_{1} \xi(t-1)+\ldots+c_{n_{c}} \xi\left(t-n_{c}\right) \\
& -d_{1} e(t-1)-d_{2} e(t-2) \ldots \\
& -d_{n_{d}} e\left(t-n_{d}\right) .
\end{aligned}
$$

Parameters $a_{1}, a_{2}, \ldots, a_{n_{2}}, b_{0}, \ldots, d_{n_{d}}$ are estimated using the samples $u(t), y(t), t=1,2, \ldots, n$, and given a choice for the orders $n_{a}, n_{b}, n_{c}$, and $n_{d}$. Regarding the noise signal $\xi($.$) , it is assumed that \xi(t), t=1,2, \ldots, n$ is an unknown white-noise process with zero mean and variance (unknown, but constant) $\sigma_{\xi}^{2}$ and that $\xi($.$) is$ independent of the input signal $u($.$) .$

Functions of the Program. Before the start of the estimation procedure, two optional transformations can be done on the data over the chosen estimation interval $t=1,2, \ldots, n$ : (1) A test on stragglers in the output signal $y$ is reported if the values $y(t), t=1, \ldots, n$ exceed a specified upper or lower boundary; and (2) the input signal as well as the output signal can be transformed to deviations from the mean according to $x(t)=x(t)-x$, with $x=[x(1)+\ldots+x(n)] / n$.

The orders of the model have to be specified. For $\mathrm{n}_{\mathrm{a}}, \mathrm{n}_{\mathrm{b}}, \mathrm{n}_{\mathrm{c}}$, and $\mathrm{n}_{\mathrm{d}}$, a minimal and a maximal value can be specified. Identification of the model is performed for all combinations of orders within the given bounds. Optionally, parameters to be estimated can be given a starting value.

The model identification consists of two parts: the estimation (using one of the available estimation methods) of the model parameters and the appreciation of the estimates with an estimate of the variance of the parameter estimate and an analysis of the residuals. Currently, two estimation methods can be chosen: the linear least-squares method (LS) and the extended matrix method (EMM), which is a recursive method.

The well-known LS is a one-shot estimation method. Parameter estimates and variances are calculated using all the samples $u(t), y(t), t=1,2, \ldots, n$. The method is applicable for the estimation of the parameters of the process model: $\mathrm{n}_{\mathrm{c}}=\mathrm{n}_{\mathrm{d}}=0$. 
EMM (Talmon \& van den Boom, 1973) is a recursive parameter estimation method. It can be considered as an extension of the recursive least-squares method (RLS), which only estimates the parameters of the process model $\left(n_{c}=n_{d}=0\right)$, and the recursive extended least-squares method, which, in addition to the parameters of the process model, also estimates the parameters of the moving average part of the noise model $\left(n_{d}=0\right)$ (Söderström, Ljung, \& Gustavsson, 1978). EMM gives an estimation of the parameters and an approximation of the parameter estimate variances at each instant $t$, $t=1,2, \ldots, n$ of the estimation interval.

To evaluate the goodness of the estimates, several possibilities exist. First, the standard deviations and the correlation matrix of the estimated parameters are calculated. Second, residuals are analyzed and tested for whiteness and normality. The multiple-correlation coefficient is evaluated as an indication of the fit of the estimated equation.

Structure of the Package. RECEST is written in FORTRAN IV for an RSX 11/D and IAS operating system (both DEC products).

The package consists of several modules: (1) Initial dialogue to determine whether interactive mode or batch mode is to be used. (2) Presentation of a questionnaire (for interactive mode only) and input of answers to determine which options are chosen. Starting values of the parameters are given here. (3) The estimation process itself, with either LS or EMM and the evaluation of the estimation results, including residual analysis. Results are output to a disk file. (4) A separate output production program (RECREA) that either prints the entire output file or chooses defined parts of it to provide summary information about the results of the estimation.

In our application, the interactive mode and full listing of output were used during the initial phase of the analysis. Batch mode with printout of summary informa- tion was used in the "production phase" of the analysis when several hundred time series had to be analyzed.

RECEST is a successor of SATER (van den Boom \& Lemmens, Note 1), which is a rather large package of interactive modules for signal analysis. in RECEST, only two of the estimation modules from SATER are implemented. They were extended and adapted for applications in which many time series are to be analyzed. Due to the modularity of RECEST, other estimation methods can easily be implemented if necessary.

Availability. Listings are available from the authors free of charge. Sources can be provided at a handling fee when a floppy-diskette (RX01 compatible, single density, single sided) or a formatted RK05 disk is sent.

\section{REFERENCE NOTE}

1. van den Boom, A. J. W., \& Lemmens, W. J. M. SATER: An interactive program package for education and research in parameter estimation, control and signal analysis techniques. Paper presented at the IFAC Symposium on Trends in Automatic Control Education, Barcelona, 1977.

\section{REFERENCES}

Hays, W. L. Statistics for the social sciences (2nd ed.). New York: Holt, Rinehart, \& Winston, 1973.

Södenström, T., Luung, L., \& Gustaysson, I. A theoretical analysis of recursive identification methods. Automatica, 1978, 14, 231-244.

Talmon, J. L., \& van den Boom, A. J. W. On the estimation of the transfer function parameters of process and noise dynamics using a single-stage estimator. In P. Eykhoff (Ed.), Proceedings of the Third IFAC Symposium on Identification and System Parameter Estimation. The Hague: Delft, 1973.

van Busser, F. J. J. Human prediction of time series. IEEE Transactions on Systems, Man, and Cybernetics, in press.

(Accepted for publication June 16, 1980.) 